\title{
Long-term incidence and outcomes of obesity-related peripheral vascular disease after bariatric surgery
}

\author{
Osama Moussa ${ }^{1} \cdot$ Maddalena Ardissino ${ }^{2,4}$ (D) Silvia Muttoni ${ }^{2} \cdot$ Ara Faraj $^{2} \cdot$ Alice Tang $^{2} \cdot$ Omar Khan $^{3} \cdot$ Peter Collins $^{5} \cdot$ \\ Usman Jaffer $^{6}$. Sanjay Purkayastha ${ }^{1}$
}

Received: 8 August 2020 / Accepted: 15 December 2020 / Published online: 12 January 2021

(C) The Author(s) 2021

\begin{abstract}
Background and aims Patients with obesity are at high risk of suffering from arterial and venous peripheral vascular disease (PVD). Bariatric surgery is an effective strategy to achieve weight reduction for patients with obesity. The long-term impact of bariatric surgery on obesity-related morbidity is subject to increasing research interest. This study aimed to ascertain the impact of bariatric surgery on the long-term occurrence of PVD in patients with obesity.

Methods The study population was extracted from the Clinical Practice Research Datalink, a nation-wide database containing primary and secondary care records of consenting patients. The intervention cohort was 2959 patients who had undergone bariatric surgery during follow-up; their controls were 2959 propensity-score-matched counterparts. The primary endpoint was development of any PVD: arterial or venous. Secondary endpoints were incident peripheral arterial disease alone, incident peripheral venous disease alone.

Results Three hundred forty-six patients suffered a primary endpoint during follow-up. Bariatric surgery did not improve peripheral vascular disease rates as a whole, but it was associated with significantly lower event rates of arterial disease $(\mathrm{HR}=$ $0.560,95 \% \mathrm{CI} 0.327-0.959, p=0.035)$ but higher event rates of venous disease (HR $=1.685,95 \% \mathrm{CI} 1.256-2.262, p<0.001)$. Conclusions Bariatric surgery was associated with significantly reduced long-term occurrence of arterial disease but increased occurrence of venous disease in patients with obesity.
\end{abstract}

Keywords Peripheral vascular disease $\cdot$ Venous insufficiency $\cdot$ Arterial disease $\cdot$ Ischaemia $\cdot$ Bariatric surgery $\cdot$ Obesity

$\overline{\text { Osama Moussa and Maddalena Ardissino are first co-authors with equal }}$ contribution

Maddalena Ardissino

ma5713@ic.ac.uk

1 Department of Surgery and Cancer, Imperial College London, Praed Street, London W2 1NY, UK

2 Department of Medicine, Imperial College London, London SW7 2AZ, UK

3 Department of Upper GI and Bariatric Surgery, St George's University of London, London SW17 0RE, UK

4 Academic Unit, Imperial College London School of Medicine, Exhibition Road, London SW7 2AZ, UK

5 Royal Brompton Hospital and National Heart and Lung Institute, Imperial College London, London SW3 6NP, UK

6 Department of Vascular Surgery, St Mary's Hospital, Imperial College NHS Trust, Praed Street, London W2 1NY, UK

\section{Introduction}

Since the beginning of its clinical development and application in the 1950s, bariatric surgery has steadily grown in popularity as a treatment option for patients with obesity [1]. In recent years, its use experienced a further surge that can be broadly related to three main reasons. Firstly, the development of endoscopic and minimally invasive techniques has allowed faster and safer intervention with lower risk of complications. Secondly, an ever-increasing volume of data has validated it as an extremely effective method of achieving weight reduction and, perhaps most importantly, of controlling some of its most severe and life-threating comorbidities such as hypertension, dyslipidaemia, and diabetes [2-4]. Thirdly, the worldwide rates of obesity are steeply rising, creating a truly global epidemic, and causing unprecedented rates of obesity-related morbidity and mortality [5].

The detrimental health effects of obesity are numerous and have been previously extensively explored. Vascular 
complications are among the most common, including peripheral arterial and venous disease. The correlation between obesity and arterial vascular morbidity may be mediated by the increased inflammation [6] and increased incidence of risk factors for atherosclerosis which are associated with obesity: type 2 diabetes, hypertension, and dyslipidaemia. Bariatric surgery is known to improve the control of, and even induce resolution of metabolic and atherosclerotic vascular risk factors, [3, 7-9] suggesting a potential role in the reduction of long-term risk of arterial disease. With regards to venous disease, the potential haemodynamic effects of decreased intra-abdominal pressure [10, 11], secondary to weight loss from bariatric surgery, may feasibly improve venous return and therefore decrease the risk of lower limb venous insufficiency and thrombosis. For these reasons, it is reasonable to hypothesize that beyond its effect on weight, bariatric surgery may be beneficial in reducing the risk of peripheral arterial and lower limb venous morbidity in patients with obesity.

Despite the established correlation between obesity and both peripheral arterial and lower limb venous disease, this clinical question has not previously been addressed. The aim of this nation-wide cohort study is to ascertain the impact of bariatric surgery on the long-term incidence of peripheral arterial and lower limb venous morbidity in patients with obesity.

\section{Methods}

\section{Study database}

The data analysed in this study was acquired from the Clinical Practice Research Datalink (CPRD) database, a UK-based governmental research database funded by the National Institute for Health Research (NIHR) and the Department of Health (DoH). Informed consent was obtained from all individual participants included in the study. The data contained within CPRD is anonymised and continuously updated, and has been demonstrated to be an accurate representation of the British population in terms of ethnicity and demographics $[12$, 13]. The design, implementation, and maintenance of this database have been previously described $[13,14]$.

\section{Ethical approval}

Scientific approval for this study was granted by The Regulatory Agency's Independent Scientific Advisory Committee (ISAC approval registration number: 16_140R2). Ethical approval was granted by the Health Research Authority IRAS. Project ID: 203143.

\section{Patient population}

A retrospective, nested cohort study was designed. Inclusion criteria was limited to the 231,389 patients that had a recorded body mass index (BMI) of $\geq 30$ $\mathrm{kg} / \mathrm{m}^{2}$ in their healthcare record before cohort inclusion. A total of 14,715 patients were excluded from the analysis due to insufficient follow-up time to establish longterm outcomes ( $<6$ months), missing information on BMI, gender, or age. Patients who had a pre-existing diagnosis of peripheral arterial or lower limb venous disease before enrollment in either study arm were also excluded. This resulted in a total of 216,674 patients considered for analysis. Bariatric surgery patients were then matched on $\mathrm{s}$ 1:1 basis with equal numbers of control on the basis of a propensity score that included all baseline demographic (age, gender, BMI) and morbidity (hyperlipidaemia, hypercholesterolaemia, diabetes, atrial fibrillation, smoking) variables. A total of 2959 bariatric surgery patients were successfully matched with a caliper of 0.05 , and 987 could not be matched due to missing information (the patient selection process is outlined in Fig. 1.

The study variables were defined as follows. Gender refers to the gender of the participants as coded in the demographic information in the database. Age refers to the age at the time of index date (the year of bariatric surgery in the intervention cohort, and the year of entry to the database for the control cohort). Baseline BMI reading refers to the BMI reading closest in time to the date of indexing.

Demographic details of all included patients were noted, specifically the baseline BMI at the time of first diagnosis of obesity, gender, age, the presence of several morbidity, and treatment factors at the time of index date (hypertension, hyperlipidaemia, diabetes mellitus, atrial fibrillation, smoking, alcohol) as well as the use of a number of medications (warfarin, direct oral anticoagulants, aspirin, beta blockers, angiotensinconverting enzyme inhibitors).

\section{Endpoint definition}

The primary endpoint of this study was a composite of occurrence of any peripheral arterial disease or peripheral venous disease.

Secondary endpoints included the individual evaluation of occurrence of the following:

- Peripheral arterial disease alone

- Peripheral venous disease alone

Peripheral arterial disease was defined as the presence of any of the following diagnoses: claudication, arterial 
Fig. 1 Patient selection flowchart

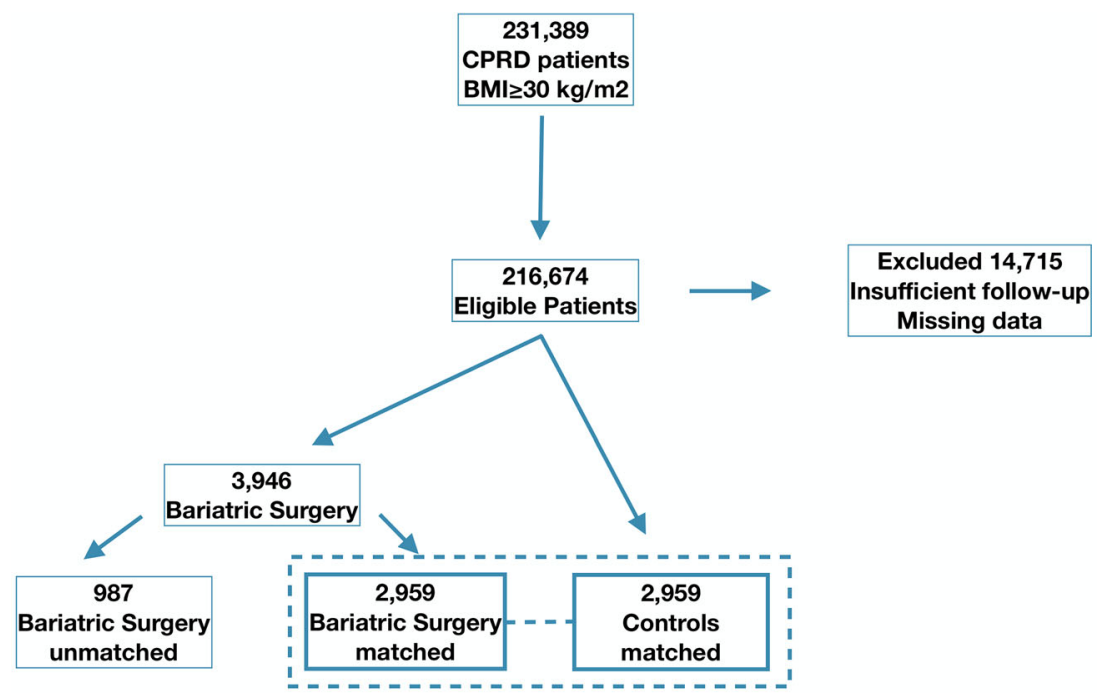

insufficiency, acute or chronic limb ischaemia, arterial ulcers or a recorded $\mathrm{ABPI}<0.8$.

Peripheral venous disease was defined by the presence of a diagnosis of venous insufficiency, venous ulcers, venous eczema, or varicose veins.

Repeat events were not included in the analysis for either primary or secondary endpoints. Both binary and time-to-event data were extracted for all outcomes. Event rates are expressed in both overall percentages, and in rates per 10,000 person-years (/10000 py) in view of the different lengths of follow-up across cohorts.

\section{Statistical analysis}

Statistical Package for Social Sciences, version 25 was used for all data extraction and preparation (SPSS Inc., New York, NY, USA) [15]. The data for the study was analysed using RStudio (Version 1.2.5033 (C) 2009-2019 RStudio, Inc.).

Baseline demographic, clinical, and treatment factors were compared between study arms using the Pearson's chi-squared test for categorical variables. Hazard data was analysed for both primary and secondary endpoints using a Cox proportional hazards model that was adjusted for multiple covariates. All factors found to be imbalanced across cohorts were adjusted for in the models. Interaction analysis was performed using a Cox proportional hazards model with interaction terms. Two sensitivity analyses were performed. Firstly, one without any covariate adjustment. Secondly, one including only patients who underwent gastric band or bypass as their bariatric surgery.

All $p$ values reported are 2-sided; and statistical significance was considered when $p<0.05$.

\section{Results}

\section{Baseline characteristics}

Five thousand nine hundred and eighteen $(5,918)$ were included in the study. Of these, 2,959 had undergone bariatric surgery and 2,959 propensity-score-matched controls had not. The mean length of total follow-up for all patients was 91.6 months $(52.7 \pm 39.8$ bariatric surgery cohort, $130.3 \pm 85.6$ controls) equating to 7.6 years. The propensity score density plots before and after matching are depicted in Supplementary Figure 1.

The mean age at first recorded diagnosis of obesity was 44.7 (44.6 \pm 10.6 bariatric surgery cohort, $44.8 \pm 13.1$ controls). Patients who underwent bariatric surgery achieved significantly more weight loss during follow-up compared to their matched controls $(p<0.001)$.

The baseline demographic, clinical, and treatment characteristics of patients by cohort are described in Table 1 .

\section{Primary endpoint}

The primary endpoint of all types of peripheral vascular disease (arterial disease, venous disease) occurred in a total of 346 patients. Patients in the bariatric surgery group had similar event rates $(\mathrm{HR}=1.223,95 \% \mathrm{CI} 0.947-1.580, p=0.124)$. The results of the analysis are outlined in Table 2 and depicted in Fig. 2.

\section{Secondary endpoint}

A total of 122 patients had a diagnosis of peripheral arterial disease during follow-up; a total of 243 patients developed lower limb venous disease (Fig. 3). 
Table 1 Baseline demographic, clinical, and treatment characteristics of the patients divided by surgery status

\begin{tabular}{|c|c|c|c|}
\hline Characteristics & No bariatric surgery $(n=2959)$ & Bariatric surgery $(n=2959)$ & $p=$ \\
\hline \multicolumn{4}{|l|}{ Demographic } \\
\hline Age (mean, SD) & $44.8(13.1)$ years & $44.6(10.6)$ years & 0.311 \\
\hline BMI (mean, SD) & $41.2(9.1) \mathrm{kg} / \mathrm{m}^{2}$ & $41.2(7.2) \mathrm{kg} / \mathrm{m}^{2}$ & 0.371 \\
\hline Male & $506(17.1 \%)$ & $645(21.8 \%)$ & $<0.001$ \\
\hline$\Delta$ Weight during follow-up (mean, SD) & $1.4(14.0) \mathrm{kg}$ & $-24.5(25.3) \mathrm{kg}$ & $<0.001$ \\
\hline \multicolumn{4}{|l|}{ Clinical } \\
\hline Hypertension $(n, \%)$ & $773(26.1 \%)$ & $861(29.1 \%)$ & 0.011 \\
\hline Resolved $(n, \%)$ & $67 / 773(8.7 \%)$ & $80 / 861(9.3 \%)$ & 0.316 \\
\hline Hyperlipidaemia $(n, \%)$ & $161(5.4 \%)$ & $194(6.6 \%)$ & 0.080 \\
\hline Resolved $(n, \%)$ & $27 / 161(16.8 \%)$ & $49194(25.3 \%)$ & 0.015 \\
\hline Diabetes $(n, \%)$ & $654(22.1 \%)$ & $750(25.4 \%)$ & 0.004 \\
\hline Resolved $(n, \%)$ & $34 / 654(5.2 \%)$ & $39 / 750(5.2 \%)$ & 0.638 \\
\hline Atrial Fibrillation $(n, \%)$ & $1(0.03 \%)$ & $25(0.8 \%)$ & $<0.001$ \\
\hline Smoking $(n, \%)$ & $1079(36.5 \%)$ & $1076(36.4 \%)$ & 0.957 \\
\hline Alcohol $(n, \%)$ & $422(14.3 \%)$ & $539(18.2 \%)$ & $<0.001$ \\
\hline \multicolumn{4}{|l|}{ Treatment } \\
\hline Warfarin $(n, \%)$ & $176(6.0 \%)$ & $125(4.2 \%)$ & 0.003 \\
\hline Direct oral anticoagulant $(n, \%)$ & $26(0.88 \%)$ & $25(0.84 \%)$ & 1.000 \\
\hline Aspirin $(n, \%)$ & $166(5.6 \%)$ & $783(26.5 \%)$ & $<0.001$ \\
\hline Statin $(n, \%)$ & $166(5.6 \%)$ & $783(26.5 \%)$ & $<0.001$ \\
\hline ACE inhibitors $(n, \%)$ & $44(1.5 \%)$ & $280(9.5 \%)$ & $<0.001$ \\
\hline B-Blockers (n,\%) & $187(6.3 \%)$ & $482(16.3 \%)$ & $<0.001$ \\
\hline Oral Hypoglycaemic (n,\%) & $221(7.5 \%)$ & $729(24.6 \%)$ & 0.000 \\
\hline \multicolumn{4}{|l|}{ Follow-up } \\
\hline Follow-up (mean, SD) & 130.3 (85.6) months & 52.7 (39.8) months & $<0.001$ \\
\hline
\end{tabular}

Peripheral arterial disease was experienced by 21 of the bariatric surgery patients and 101 of the controls, with rates of 1.3 and 2.6 events per 10,000 patient years respectively $(\mathrm{HR}=0.560,95 \% \mathrm{CI} 0.327-0.959, p=0.035)$. Ninety-nine in the bariatric surgery cohort and 144 of the controls received a diagnosis of lower limb venous disease during follow-up, translating to a rate of 6.3 and 3.7 events per 10,000 patient years respectively $(\mathrm{HR}=1.685,95 \% \mathrm{CI} 1.256-2.262, p<$ $0.001)$. The results of the analyses are displayed in Table 2.

\section{Sensitivity and subgroup analyses}

Two sensitivity analyses were carried out. Firstly, without adjustment for covariates and secondly only including patients who had gastric bypass and gastric banding. Both analyses yielded similar results to primary analysis (Table 3 ).

Interaction analysis revealed a stronger effect size in patients of higher BMI classes at index $(p=0.229)$; but no significant interaction with gender was present $(p=0.384)$ as displayed in Table 3. There was no difference in rates of primary events across different bariatric surgery types ( $p=$ 0.767), as displayed in Table 4.

\section{Discussion}

Bariatric surgery is a growing field that is rapidly becoming a major cornerstone in the management of patients with obesity. Its use in the UK has grown precipitously over the last decade: in a recent epidemiological study based on CPRD data, the number of surgeries performed in the 2010-2012 interval was found to be more than nine times higher than in the 20022005 interval. However, despite its growing use, much remain unanswered with regards to its long-term clinical impact. Does it truly reduce obesity-related morbidity in the longterm or does it only provide temporary benefit? Do the potential long-term benefits outweigh the short-term risks of the surgical procedure and cost?

Because of its relatively short history, evidence on the long-term effects of the surgery is currently still scarce, with only a handful of promising studies achieving adequate cohort sizes and long follow-up time. This study presents data that adds to this growing pool of evidence with one of the largest bariatric cohorts reported on to date, addressing the lower limb arterial and venous outcomes of 2,959 patients in the CPRD. The results of the study reveal similar overall rates of 
Table 2 Endpoint occurrence during follow-up

\begin{tabular}{|c|c|c|c|c|c|}
\hline Events (per 10,000 person-years) & $\begin{array}{l}\text { No bariatric surgery } \\
(n=2959\end{array}$ & $\begin{array}{l}\text { Bariatric surgery } \\
(n=2959)\end{array}$ & HR & $95 \% \mathrm{CI}$ & $p=$ \\
\hline \multicolumn{6}{|l|}{ Primary outcomes } \\
\hline Primary outcome: peripheral vascular disease ${ }^{a}(/ 10,000$ py) & 6.0 & 7.5 & 1.223 & $0.947-1.580$ & 0.124 \\
\hline Peripheral arterial disease ${ }^{\mathrm{b}}(/ 10,000$ py $)$ & 2.6 & 1.3 & 0.560 & $0.327-0.959$ & 0.035 \\
\hline Peripheral venous disease ${ }^{c}(/ 10,000$ py) & 3.7 & 6.3 & 1.685 & $1.256-2.262$ & $<0.001$ \\
\hline
\end{tabular}

${ }^{\text {a }}$ Peripheral vascular disease events: composite of arterial disease, and venous disease

${ }^{\mathrm{b}}$ All peripheral arterial disease outcomes: composite of claudication, angina cruris, arterial insufficiency, limb ischaemic (acute or chronic), arterial ulcer, ABPI $<0.8$

${ }^{\mathrm{c}}$ All peripheral venous disease outcomes: composite of venous ulcer, venous insufficiency, varicose veins, venous eczema

peripheral vascular morbidity but reveal a lower rate of arterial disease among the bariatric cohort which is offset by higher rates of venous disease. Considering the high vascular morbidity that is associated with obesity, and the relative gravity of arterial disease when compared to venous disease, the potential beneficial effect of bariatric surgery on arterial outcomes reported in this study may translate to an important clinical benefit in the current obesity epidemic.

There are a number of possible interpretations for the study results. Firstly, the reduced rate of peripheral arterial disease detected in the bariatric surgery patients is likely to relate to an improvement in risk factor control. As evidenced by the higher rates of resolution of risk factors in the bariatric surgery cohort, and supported by prior literature on the subject $[3,16]$, this is the most likely underlying explanation. This result is in concordance with previous literature that has detected lower rates of atherosclerotic disease in a number of bariatric surgery cohorts, in the context of coronary, and other macrovascular diseases [17, 18]. A further potential contributing mechanism for this is that bariatric surgery is known to be associated with

\section{Primary Composite Endpoint}

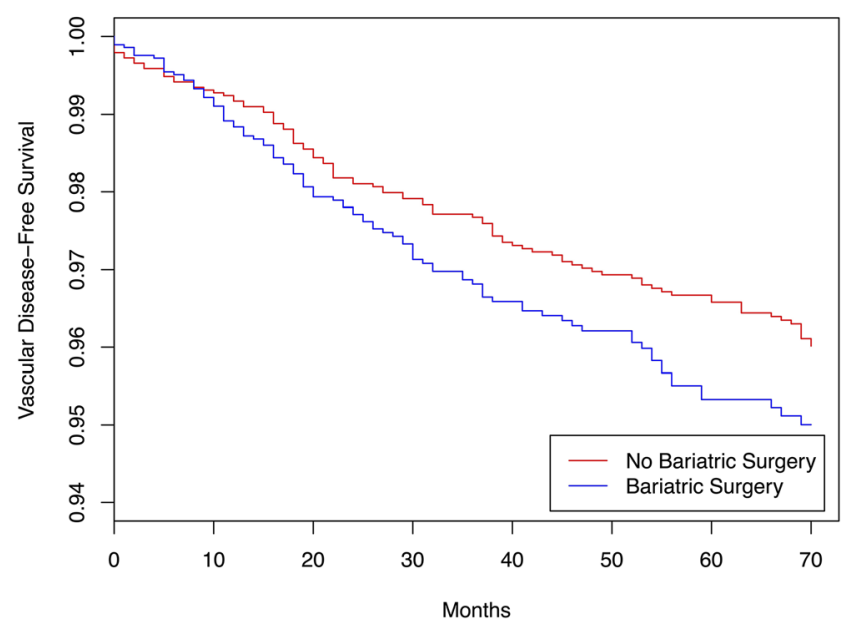

Fig. 2 Adjusted primary endpoint rates by bariatric surgery status during follow-up time lower rates of incident $\mathrm{AF}$ and higher rates of $\mathrm{AF}$ resolution $[19,20]$, which may contribute to lower arterial thrombosis rates.

On the other hand, the rates of venous disease were higher among the bariatric surgery cohort. This goes against the hypothesis we provided for our study, whereby lower intra-
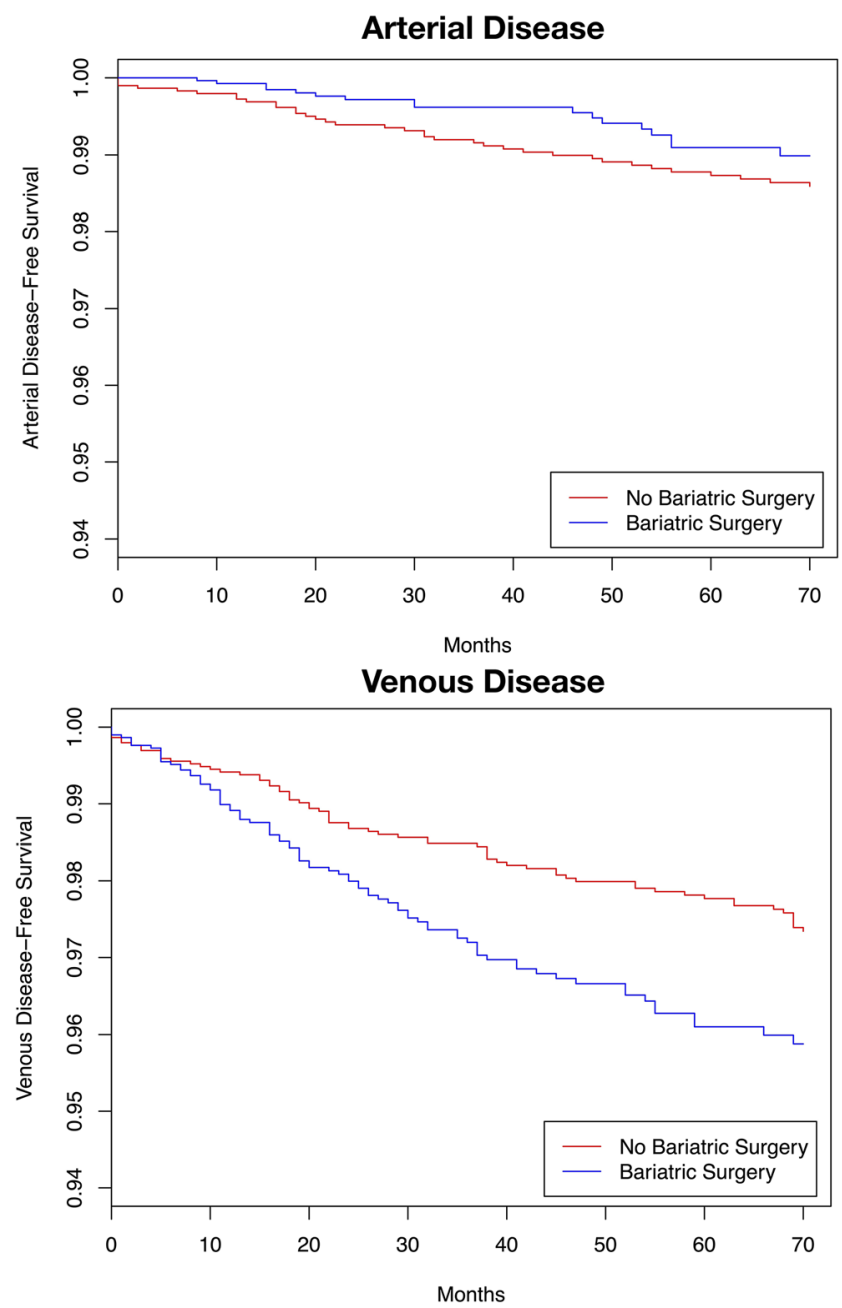

Fig. 3 Adjusted secondary endpoint rates by bariatric surgery status during follow-up time 
Table 3 Sensitivity analysis for sleeve gastrectomy and gastric bypass surgery

\begin{tabular}{lccc}
\hline & HR & $95 \%$ CI & $p=$ \\
\hline Sensitivity model 1: unadjusted model & & & \\
Primary outcome: peripheral vascular disease $^{\mathrm{a}}$ & 1.190 & $0.932-1.520$ & 0.164 \\
Peripheral arterial disease $^{\mathrm{b}}$ & 0.584 & $0.354-0.962$ & 0.035 \\
Peripheral venous disease $^{\mathrm{c}}$ & 1.603 & $1.207-2.129$ & 0.001 \\
Sensitivity model 2: including sleeve gastrectomy and gastric bypass only & & \\
Primary outcome: peripheral vascular disease $^{\mathrm{a}}$ & 1.026 & $0.734-1.434$ & 0.881 \\
Peripheral arterial disease $^{\mathrm{b}}$ & 0.336 & $0.133-0.847$ & 0.021 \\
Peripheral venous disease $^{\mathrm{c}}$ & 1.380 & $0.961-1.981$ & 0.081 \\
\hline
\end{tabular}

${ }^{a}$ Peripheral vascular disease events: composite of arterial disease, and venous disease

${ }^{\mathrm{b}}$ All peripheral arterial disease outcomes: composite of claudication, angina cruris, arterial insufficiency, limb ischaemic (acute or chronic), arterial ulcer, ABPI $<0.8$

${ }^{\mathrm{c}}$ All peripheral venous disease outcomes: composite of venous ulcer, venous insufficiency, varicose veins, venous eczema abdominal pressure was thought to have the potential to contribute to better venous return and therefore lower rates of peripheral venous disease. There are a number of potential explanations for this discrepancy. Firstly, and most importantly, it is likely that this endpoint is subject to a degree of measurement bias. As patients who undergo bariatric surgery are subject to stricter and more frequent follow-up, the increase in medical contact provides higher opportunity to pick up symptoms from venous disease. Secondly, it is possible that the reduction in mobility perioperatively, and concordant risk of venous thrombosis also plays a role in the increased rates of venous disease. However, we would expect this impact to be minor in view of the long follow-up time in this study.

\section{Limitations}

Due to data coding reasons, it was not possible for us to discern amputations secondary to lower limb vascular disease, as opposed to those due to traumatic, infectious, malignant or other causes. For this reason, amputation was not considered as an endpoint in this study. Similarly, we are unable to determine the exact symptomology that clinically coded diagnoses correspond to, or extract information regarding specific interventions performed for PAD. However, considering the primary endpoint is the occurrence of new $\mathrm{PAD}$, this rate should be shielded from impact. Further evaluation, in the context of a database specifically designed to investigate and distinguish the rates of lower limb vascular disease-related amputations may be warranted. Furthermore, due to lack of data granularity, it was not possible to accurately establish dosing and timing of medication use among patients. This is an important limitation, and further assessment in a database that contains suitable detail for this purpose is warranted.

The CPRD has been previously widely validated as being accurately representative of the UK population for demographic characteristics such as age, sex, morbidities, and ethnicity. This implies that the results of this study are likely to be generalisable to any Western country that has similar demographic characteristics to the UK. A benefit of this study is the large number of patients included, which improves the reliability of the data and the conclusions that are drawn from it. However, its design also presents some limitations. The medical records used for the database are totally anonymised, and it is therefore not feasible to fill or research missing follow-up data. The follow-up is therefore strictly limited to permanence in the database which is in part geographically determined.
Table 4 Primary outcome occurrence by bariatric surgery type

\begin{tabular}{lllc}
\hline Bariatric surgery type & $\begin{array}{l}\text { Number of patients } \\
(n=2959)(n)\end{array}$ & $\begin{array}{l}\text { Number of patients with } \\
\text { primary events }(n, \%))\end{array}$ & $p=($ logrank $)$ \\
\hline Gastric banding & 1147 & $51(4.4 \%)$ & 0.767 \\
Gastric bypass & 1207 & $39(3.23 \%)$ & \\
Sleeve gastrectomy & 432 & $6(1.38 \%)$ & \\
Duodenal switch & 21 & 0 \\
Gastric stapling & 7 & $2(28.5 \%)$ \\
Stomach partitioning & 5 & 0 \\
Mason vertical banded gastroplasty & 4 & 0 \\
Undefined bariatric surgery & 136 & $17(12.5 \%)$
\end{tabular}


Furthermore, as the data collection is linked to primary- and secondary-care diagnostic data, its accuracy is entirely reliant on the accuracy of the clinicians' diagnosis, and on the accuracy of data coding and transcription into the database. Additionally, the CPRD population with obesity is predominantly female; this has implications for the external validity of the study allowing the results to be generalisable more to females than to males. In order to reduce the potential bias that this may have, we have matched the cohorts on variables including sex.

\section{Conclusion}

In conclusion, this study provides evidence on the long-term lower limb vascular morbidity of patients with obesity who undergo bariatric surgery. While we did not observe a significant protective effect of bariatric surgery on peripheral vascular disease as a whole, we did find a significant reduction in arterial morbidity in patients who underwent bariatric surgery when compared to propensity-score-matched controls that did not. In contrast, we also noted a significant increase in venous disease in bariatric surgery patients, which may relate to an increased rate of detection due to the higher degree of contact with routine care in patients who undergo the surgery. The findings of the study are important both for immediate clinical practice and to guide further research. With the wealth of emerging evidence regarding the benefits of bariatric surgery, that range from mental health to the resolution of diabetes, the indications for bariatric surgery, and clinician awareness of its benefits is growing, but evidence still has a long way to go. These results highlight the requirement for a large-scale, randomised controlled trial investigating the long-term effect of bariatric surgery, in order to validate the emerging results from retrospective studies. Furthermore, the issue of takeup must be considered: less than $1 \%$ of patients who are eligible for bariatric surgery currently undergo it. Considering the potential benefits displayed in this study, combined with the wealth of evidence that suggests reductions in cancer-related mortality, diabetes, all-cause mortality and many more, the lack of access to care experienced by the $99 \%$ of eligible patients may represent avoidable, long-term adverse clinical outcomes. Overall, it is time to consider the factors that are contributing to this unmet clinical need, in the interest of improving the long-term outcomes of patients with obesity.

Supplementary Information The online version contains supplementary material available at https://doi.org/10.1007/s00423-020-02066-9.

Acknowledgements Data access was provided by the Big Data \& Analytical Unit (BDAU) at the Institute of Global Health Innovation (IGHI), Imperial College London.

Infrastructure support for this research was provided by the NIHR Imperial Biomedical Research Centre (BRC).
Authors' contributions O.M.: conceptualisation, investigation, data analysis, manuscript review. M.A: investigation, design, manuscript writing, data interpretation, conceptualisation. S.M.: manuscript writing, data interpretation, investigation. A.F.: manuscript writing, data interpretation, investigation. A.T:. manuscript writing, study design, investigation. O.K.: supervision, manuscript review, data interpretation. P.C.: manuscript review, supervision, data interpretation. U.J.: manuscript review, supervision, data interpretation. S.P.: data curation, resources, manuscript review, supervision.

\section{Compliance with ethical standards}

Conflict of interest The authors declare that they have no conflicts of interest.

Ethical approval All procedures performed in studies involving human participants were in accordance with the ethical standards of the institutional and/or national research committee and with the 1964 Helsinki declaration and its later amendments or comparable ethical standards.

Informed consent Informed consent was obtained from all individual in accordance with the procedure of the Clinical Practice Research Datalink.

Open Access This article is licensed under a Creative Commons Attribution 4.0 International License, which permits use, sharing, adaptation, distribution and reproduction in any medium or format, as long as you give appropriate credit to the original author(s) and the source, provide a link to the Creative Commons licence, and indicate if changes were made. The images or other third party material in this article are included in the article's Creative Commons licence, unless indicated otherwise in a credit line to the material. If material is not included in the article's Creative Commons licence and your intended use is not permitted by statutory regulation or exceeds the permitted use, you will need to obtain permission directly from the copyright holder. To view a copy of this licence, visit http://creativecommons.org/licenses/by/4.0/.

\section{References}

1. Baker MT (2011) The history and evolution of bariatric surgical procedures. Surg Clin North Am 91(6):1181-1201. https://doi.org/ 10.1016/j.suc.2011.08.002

2. Douglas IJ, Bhaskaran K, Batterham RL, Smeeth L (2015) Bariatric surgery in the United Kingdom: a cohort study of weight loss and clinical outcomes in routine clinical care. PLoS Med 12(12):1-18. https://doi.org/10.1371/journal.pmed.1001925

3. Mistry P, Currie V, Super P, le Roux CW, Tahrani AA, Singhal R (2018) Changes in glycaemic control, blood pressure and lipids 5 years following laparoscopic adjustable gastric banding combined with medical care in patients with type 2 diabetes: a longitudinal analysis. Clin Obes 8:151-158. https://doi.org/10.1111/cob.12244

4. Sjöström L, Peltonen M, Jacobson P, Ahlin S, AnderssonAssarsson J, Anveden Å, Bouchard C, Carlsson B, Karason K, Lönroth H, Näslund I, Sjöström E, Taube M, Wedel H, Svensson PA, Sjöholm K, Carlsson LMS (2014) Association of bariatric surgery with long-term remission of type 2 diabetes and with microvascular and macrovascular complications. JAMA - J Am Med Assoc 311:2297-2304. https://doi.org/10.1001/jama.2014.5988

5. WHO (2011) WHO | Obesity and overweight. World Heal Organ Media Cent Fact Sheet No 311, pp 1-2. https://doi.org/10.3233/ 978-1-61499-438-1-372

6. Aghamohammadzadeh R, Greenstein AS, Yadav R, Jeziorska M, Hama S, Soltani F, Pemberton PW, Ammori B, Malik RA, Soran 
H, Heagerty AM (2013) Effects of bariatric surgery on human small artery function: evidence for reduction in perivascular adipocyte inflammation, and the restoration of normal anticontractile activity despite persistent obesity. J Am Coll Cardiol 62:128-135. https:// doi.org/10.1016/j.jacc.2013.04.027

7. Jakobsen GS, Småstuen MC, Sandbu R, Nordstrand N, Hofsø D, Lindberg M, Hertel JK, Hjelmesæth J (2018) Association of bariatric surgery vs medical obesity treatment with long-term medical complications and obesity-related comorbidities. JAMA. 319: 291-301. https://doi.org/10.1001/jama.2017.21055

8. Benotti PN, Wood GC, Carey DJ, Mehra VC, Mirshahi T, Lent MR, Petrick AT, Still C, Gerhard GS, Hirsch AG (2017) Gastric bypass surgery produces a durable reduction in cardiovascular disease risk factors and reduces the long-term risks of congestive heart failure. J Am Heart Assoc 6. https://doi.org/10.1161/JAHA.116. 005126

9. Poirier P, Cornier MA, Mazzone T, Stiles S, Cummings S, Klein S, McCullough P, Ren Fielding C, Franklin BA, American Heart Association Obesity Committee of the Council on Nutrition, Physical Activity, and Metabolism (2011) Bariatric surgery and cardiovascular risk factors: a scientific statement from the American Heart Association. Circulation. 123:1683-1701. https:// doi.org/10.1161/CIR.0b013e3182149099

10. Willenberg T, Schumacher A, Amann-Vesti B, Jacomella V, Thalhammer C, Diehm N, Baumgartner I, Husmann M (2010) Impact of obesity on venous hemodynamics of the lower limbs. J Vasc Surg 52:664-668. https://doi.org/10.1016/j.jvs.2010.04.023

11. Beebe-Dimmer JL, Pfeifer JR, Engle JS, Schottenfeld D (2005) The epidemiology of chronic venous insufficiency and varicose veins. Ann Epidemiol 15:175-184. https://doi.org/10.1016/j.annepidem. 2004.05.015

12. Bhaskaran K, Forbes HJ, Douglas I, Leon DA, Smeeth L (2013) Representativeness and optimal use of body mass index (BMI) in the UK Clinical Practice Research Datalink (CPRD). BMJ Open 3: e003389. https://doi.org/10.1136/bmjopen-2013-003389

13. Herrett E, Gallagher AM, Bhaskaran K, Forbes H, Mathur R, van Staa T, Smeeth L (2015) Data resource profile: clinical practice research datalink (CPRD). Int J Epidemiol 44:827-836. https:// doi.org/10.1093/ije/dyv098
14. Clinical Practice Research Datalink. CPRD Governance. Clinical Practice Research Datalink website. https://www.cprd.com/ governance/. Published 2016.

15. IBM. IBM SPSS Statistics Software for Windows, Version 25. IBM. https://doi.org/10.1016/j.jchf.2014.02.009

16. Mingrone G, Panunzi S, De Gaetano A et al (2015) Bariatricmetabolic surgery versus conventional medical treatment in obese patients with type 2 diabetes: 5 year follow-up of an open-label, single-centre, randomised controlled trial. Lancet. 386:964-973. https://doi.org/10.1016/S0140-6736(15)00075-6

17. Fisher DP, Johnson E, Haneuse S, Arterburn D, Coleman KJ, O'Connor PJ, O'Brien R, Bogart A, Theis MK, Anau J, Schroeder EB, Sidney S (2018) Association between bariatric surgery and macrovascular disease outcomes in patients with type 2 diabetes and severe obesity. JAMA - J Am Med Assoc. 320:1570 1582. https://doi.org/10.1001/jama.2018.14619

18. Moussa O, Ardissino M, Heaton T, Tang A, Khan O, Ziprin P, Darzi A, Collins P, Purkayastha S (2020) Effect of bariatric surgery on long-term cardiovascular outcomes: a nationwide nested cohort study. Eur Heart J 41:2660-2667. https://doi.org/10.1093/eurheartj/ ehaa069

19. Moussa O, Ardissino M, Eichhorn C, Reddy RK, Khan O, Ziprin P, Darzi A, Collins P, Purkayastha S (2020) Atrial fibrillation and obesity: long-term incidence and outcomes after bariatric surgery. Eur J Prev Cardiol:204748732090451. https://doi.org/10.1177/ 2047487320904515

20. Wong CX, Sullivan T, Sun MT, Mahajan R, Pathak RK, Middeldorp M, Twomey D, Ganesan AN, Rangnekar G, RobertsThomson KC, Lau DH, Sanders P (2015) Obesity and the risk of incident, post-operative, and post-ablation atrial fibrillation: a metaanalysis of 626,603 individuals in 51 studies. JACC Clin Electrophysiol 1:139-152. https://doi.org/10.1016/j.jacep.2015. 04.004

Publisher's note Springer Nature remains neutral with regard to jurisdictional claims in published maps and institutional affiliations. 